
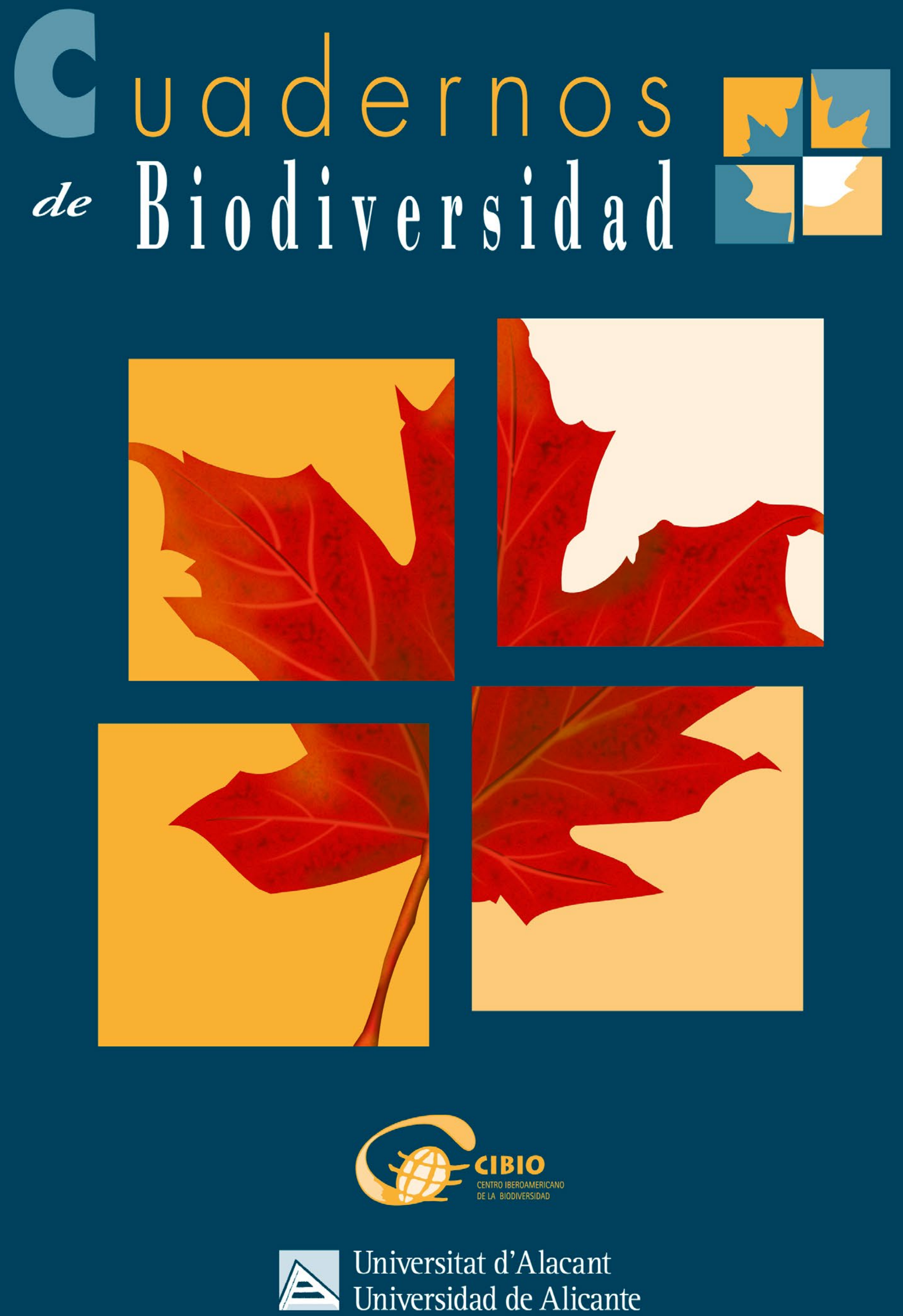


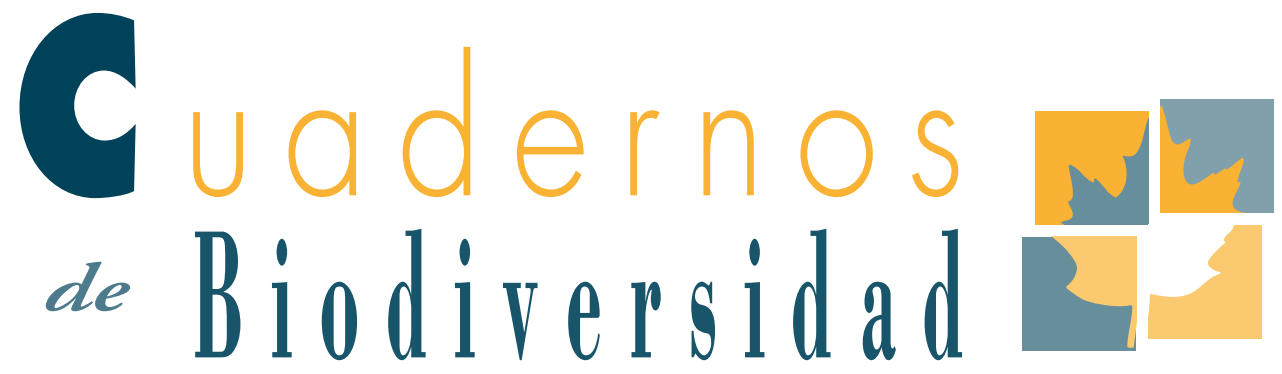

www. cuadernosdebiodiversidad.org

\section{Plan de rescate preventivo de Liolaemus confusus (Squamata: Liolaemidae), una especie en Peligro Crítico de Extinción} endémica de la Región de O'Higgins, Chile Preventive rescue plan of Liolaemus confusus (Squamata: Liolaemidae), a Critically Endangered species endemic to the O'Higgins region, Chile

\section{Ramírez-Álvarez ${ }^{*}$, I. Salgado ${ }^{1}$ y A. Montalba ${ }^{2}$.}

1 Servicio Agrícola y Ganadero SAG, Unidad de Vida Silvestre, Región de O’Higgins, Chile.

2 Zoológico Nacional de Chile, Pío Nono 450, Recoleta, Región Metropolitana, Chile.

* Correspondencia A: Diego.RAmirez@SAg.gob.Cl

\section{RESUMEN}

Se expone el plan de contingencia desarrollado por el Servicio Agrícola y Ganadero (SAG) de la Región de O’Higgins, Chile, con el apoyo del Zoológico Nacional de Chile y profesionales voluntarios, para el rescate preventivo de 20 ejemplares (10 machos y 10 hembras) de Liolaemus confusus, especie en Peligro Crítico de Extinción, desde su localidad tipo en el cerro Los Robles de la Comuna de Lolol, Región de O'Higgins, con el objetivo de preservar la especie ante la inminente amenaza de destrucción de su único hábitat y poblaciones conocidas, debido a los incendios forestales que rodearon su restringida área de distribución durante el mes de enero del 2017.

Palabras clave: Conservación, Ex - situ, Emergencia, Incendios forestales, Reptiles. 


\section{ABSTRACT}

We expose the contingency plan developed by the Chilean Agriculture and Livestock Service (SAG) O'Higgins region, with the support of the National Zoo and professional volunteers, in were 20 individuals (10 male and 10 female) of Liolaemus confusus, a Critically Endangered species, were preventive rescued from their type locality in Los Robles hills, Lolol, O'Higgins region, to preserve the species from the imminent destruction menace of their unique habitat and population, due to the forestry fire that surrounded their restricted distribution area, during January 2017.

Keywords: Conservation, emergency, forestry fire, $e x$ - situ, Reptiles.

\section{INTRODUCCIÓN}

Liolaemus confusus Núñez \& Pincheira-Donoso, 2006 (Squamata, Liolaemidae), es una especie endémica de la Región de O’Higgins, Chile. Fue descrita habitando el Cerro Los Robles de la Comuna de Lolol, Región de O'Higgins, en donde se dispuso como punto de georreferencia para su localidad tipo: $34^{\circ} 47^{\prime} 34.00^{\prime \prime} \mathrm{S} / 71^{\circ} 33^{\prime} 7.00^{\prime \prime} \mathrm{O}$ (Núñez y Pincheira-Donoso 2006), estimándose la extensión de su presencia en solo $5 \mathrm{~km}^{2}$, donde está amenazada por la degradación del suelo que genera la ganadería caprina, la corta de vegetación nativa para uso de leńa y el cambio de la cobertura vegetacional nativa por plantaciones forestales, lo que, junto a su baja abundancia en la zona, ha determinado que sea clasificada como "En Peligro Crítico de Extinción" (Ministerio del Medio Ambiente de Chile 2014). Posteriormente fueron publicadas dos nuevas georreferencias de presencia de esta especie: $34^{\circ} 45^{\prime} 52.00^{\prime \prime} \mathrm{S} / 71^{\circ} 35^{\prime} 19.52^{\prime \prime} \mathrm{O}$ y $34^{\circ} 46^{\prime} 40.27^{\prime \prime}$ S / 71³3'20.91" O, ambas en el mismo cordón de su localidad tipo, Cerro Los Robles, Lolol, calculándose, según el polígono generado, su área de extensión en 2,2 km² (González-Gutiérrez 2015). Finalmente el hallazgo de un ejemplar en el Cerro La Palma, localidad de Nerquihue, comuna de Santa Cruz, permitió ampliar en 20 kilómetros su

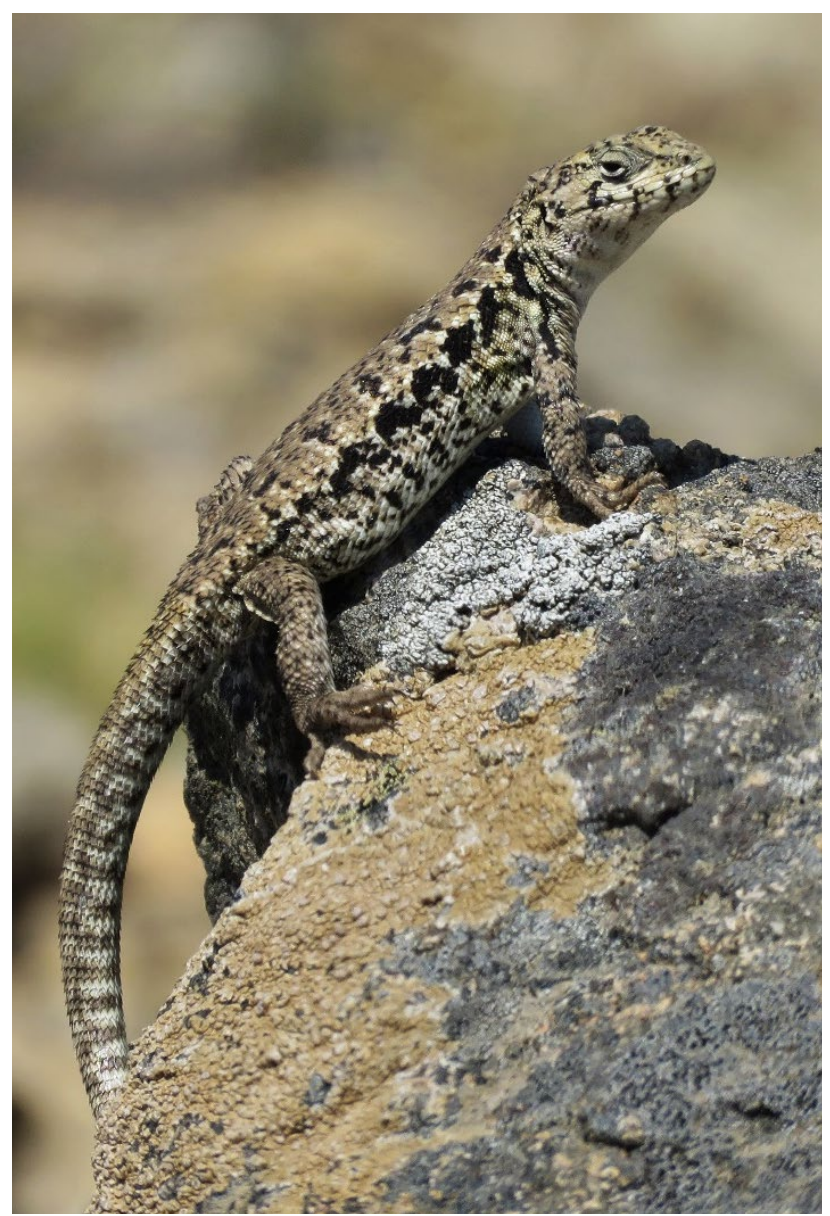

Figura 1. Liolaemus confusus

distribución lineal desde la localidad tipo, y actualizar su área de extensión a $37 \mathrm{~km}^{2}$ y su área de ocupación a $11 \mathrm{~km}^{2}$ (Ramírez-Álvarez et al. 2016). Aún considerando esta ampliación en el rango de distribución, área de extensión y ocupación para L. confusus, se ratifica la actual clasificación de su Estado de Conservación como En Peligro Crítico de Extinción, según los parámetros establecidos por la Unión Internacional para la Conservación de la Naturaleza (IUCN) y el Reglamento de Clasificación de Especies del Estado de Chile (Ministerio del Medio Ambiente de Chile 2014).

\section{INCENDIO Y AMENAZA DEL HABITAT}

Durante el mes de enero del 2017 una serie de incendios forestales de múltiples focos afectaron a la Región de O’Higgins, teniendo un origen antrópico agravado por altas velocidades de viento, altas temperaturas con sucesivas olas de calor, baja hume- 
dad y dificultosa geografía de los lugares afectados. La extensión de los territorios bajo fuego, con el subsecuente impacto social, humano, económico y ambiental, determinó la declaración por parte del Gobierno de Chile, del "Estado de excepción constitucional de catástrofe en las provincias de Colchagua y Cardenal Caro, de la Región de O'Higgins" (Ministerio del Interior y Seguridad Publica de Chile 2017) y "Zona de Emergencia Agrícola para la Región de O’Higgins” (Ministerio de Agricultura de Chile 2017a). El balance a la fecha 29 de enero del 2017, indicó que sólo en la Región de O'Higgins, 71.769 hectáreas fueron consumidas por el fuego, correspondiendo 28.800 de ellas a plantaciones forestales o terrenos de uso agrícola, y 42.969 a matorral, pradera y bosque nativo, con el correspondiente impacto sobre la biodiversidad local (Ministerio de Agricultura de Chile 2017b).

De los focos incendiaros que se desarrollaron, el denominado "Yáquil 2" afectó gran parte de la última área de distribución descrita para L. confusus, el cerro Las Palmas, de la localidad de Nerquihue, generando un daño aún incierto en esas poblaciones, y por lo tanto, sus únicas poblaciones no afectadas, volvieron a quedar circunscritas a los $5 \mathrm{~km}^{2}$ conocidos del cerro Los Robles de Lolol.

$\mathrm{El}$ avance de los incendios forestales y la generación de distintos focos cercanos determinó que, entre el 23 y el 28 de enero del 2017, el cerro Los Robles de Lolol estuviera rodeado por cuatro focos activos de fuego, denominados "Las Pataguas", hacia el este del cerro; "Nilahue-Barahona", hacia el noroeste; "El Perdigueral" hacia el oeste; y "Las Cardillas" hacia el suroeste. Este último el más agresivo, que se direccionó desde la localidad de Hualañe hacia Lolol, y cuyo perímetro de fuego alcanzó, el 26 de enero, parcialmente la ladera sur del cerro Los Robles. Dicho escenario constituyó una amenaza inminente de arrasar con las poblaciones y el único hábitat disponible conocido de L. confusus, lo cual implicaba la pérdida de un patrimonio genético y natural incalculable para la región, el país y finalmente para la diversidad biológica del planeta.

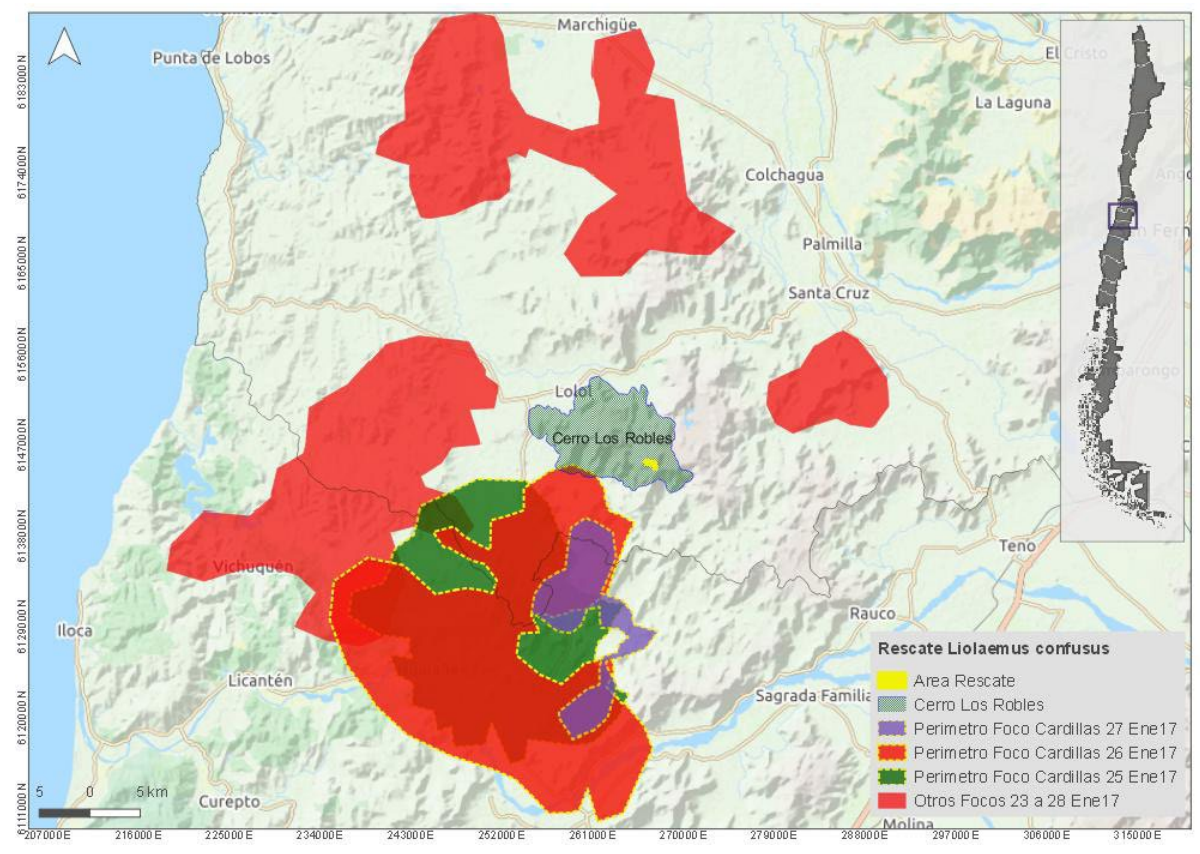

Figura 2. Escenario de los focos incendiarios que rodearon el cerro Los Robles de Lolol entre el 23 y el 28 de enero del 2017. En el centro del mapa, en verde, el perímetro del Cerro Los Robles, y dentro de él, en amarillo, el área de rescate de L. confusus. 


\section{EL PLAN DE RESCATE PREVENTIVO}

El día 24 de enero del 2017, como autoridad competente para la protección de la fauna nacional, el Servicio Agrícola y Ganadero de la Región de O'Higgins, en consulta con herpetólogos del ámbito académico nacional, generó un plan de contingencia, consistente en el rescate preventivo y mantenimiento ex - situ de un grupo de 20 ejemplares (10 machos y 10 hembras) de la especie. Si bien no existe un número concretamente definido para la cantidad de ejemplares del género Liolaemus que permitan generar una población genéticamente viable en cautiverio, sin presentar consecuencias derivadas de la endogamia y ausencia de ventaja del heterocigoto, tomamos como referencia los siguiente ejemplos: la recuperación poblacional del lagarto gigante de El Hierro (Gallotia simonyi Steindachner, 1889) en las Islas Canarias, a través de un programa de cría en cautividad y reintroducción de la especie a su hábitat natural, comenzó a partir de una estimación poblacional natural de entre 96 a 118 ejemplares (Machado 1985), siendo necesaria la optimización de cruzamientos en cautividad para reducir los efectos de la endogamia (Arano et al. 1999). Witzenberger y Hochkirch (2011) estimaron en 100 ejemplares el número adecuado para minimizar la pérdida de diversidad genética en la conservación $e x$ situ para vertebrados en general. Por otra parte, las experiencias con el Cóndor de California (Gymnogyps californianus Shaw, 1797) en Estados Unidos, se desarrollaron con un grupo inicial de 50 individuos, generándose varios manejos en el cruce para reducir el nivel de endogamia, obteniéndose hoy en día una población recuperada y genéticamente saludable (Gual 2009). Considerando el nivel evolutivo arcaico de los "Reptiles", estimamos que presentaba las condiciones adaptativas fisiológicas y genéticas para desarrollar poblaciones viables a partir de pocos individuos, y por lo tanto fijamos la cantidad a capturar en 20 ejemplares, para reducir además, el impacto ecológico sobre la población general en caso de que el hábitat no fuera afectado por el incendio.

La campaña en terreno se ejecutó el día 25 de enero del 2017, entre las 12:00 y las 16:00 horas, por un equipo de 10 personas del SAG O'Higgins, Zoo- lógico Nacional, Veterinarios y Biólogos voluntarios. Se eligieron 2 locaciones distintas en la cima del Cerro Los Robles, para obtener mayor variabilidad genética, desde cada una se capturaron 5 machos y 5 hembras. Cada individuo fue capturado a través de la técnica del lazo corredizo o manual, su punto de captura fue georreferenciado usando Datum WGS $84 \mathrm{H} 19$, y fue mantenido en caja individual de transporte con sustrato liviano (pasto seco y tierra) colectado en su mismo punto de captura.

El grupo completo de individuos fue transportado hasta las dependencias de la Dirección Regional SAG O’Higgins. Aquí fueron mantenidos en el área de subsuelo, lugar sombrío, correctamente ventilado, libre de estímulos y a relativamente baja temperatura $\left(17^{\circ} \mathrm{C}\right.$ promedio en época estival). En estas condiciones se propició la disminución del metabolismo de los ejemplares, para la conservación de sus reservas energéticas, a la espera del desarrollo de los eventos, que resolverían la situación de acuerdo a dos circunstancias:

1.- Si luego de controlados y extinguidos todos los incendios de la zona no ha habido afectación de su hábitat tipo en el Cerro Los Robles de Lolol, cada individuo sería devuelto y liberado en su punto georreferenciado de origen.

2.- Si los incendios afectaban completamente el Cerro Los Robles, y se perdía el hábitat original natural de la especie, los ejemplares serían derivados a las dependencias del Zoológico Nacional, en donde se habilitaría una zona de terrarios con manejo de todas las condiciones y variables ambientales adaptadas a la especie: Sustrato vegetacional bajo, saxícola, control de temperatura y humedad, alimentación insectívora. Posteriormente se comenzaría un manejo reproductivo con la finalidad de obtención de crías viables, las cuales, en su adecuada etapa de desarrollo, serían reintroducidas en hábitats similares al original, o en el hábitat original Cerro Los Robles, a largo plazo, cuando la cobertura vegetacional de la zona ya se hubiera recuperado. Cabe destacar que no existen experiencias a la fecha que demuestren un exitoso manejo reproductivo ex - situ de especies del género Liolaemus en Chile, por lo que esta sería una iniciativa pionera que se debería ir reevaluando y 


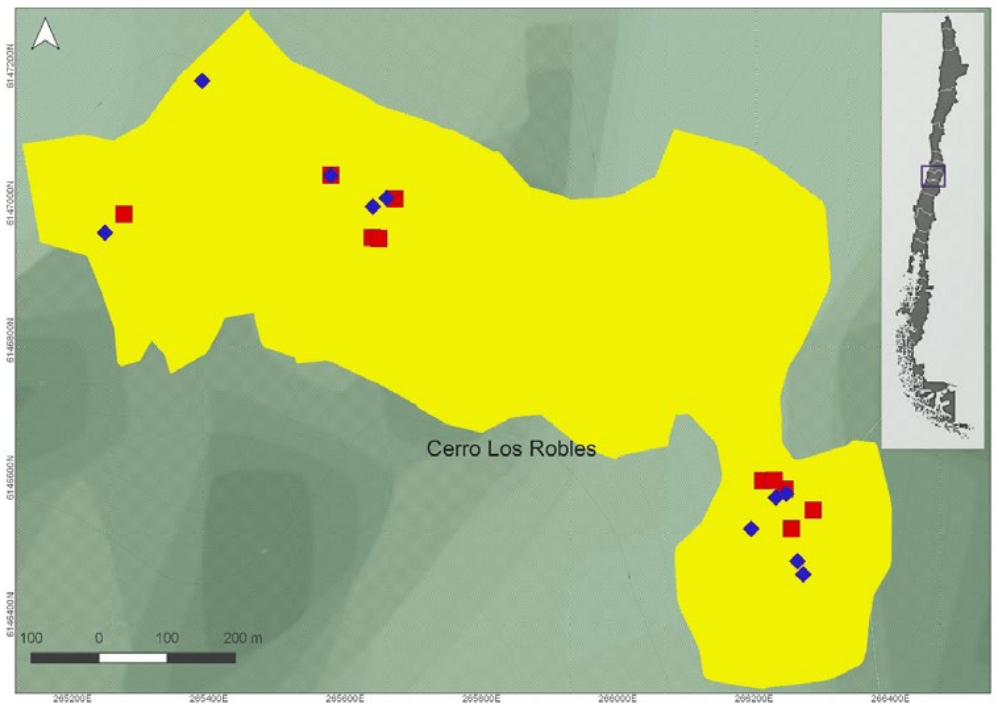

Figura 3. Distribución de los especímenes de L. confusus capturados en el área de rescate del Cerro Los Robles. Rombos azules = machos, cuadrados rojos $=$ hembras .

orientando en el transcurso del tiempo, y en función de los datos que se obtuvieran.

La incertidumbre del éxito de las medidas adoptadas quedó siempre sublimada ante la posibilidad de extinción de la especie.

Durante el periodo de cautividad y mantenimiento de los ejemplares, se realizó un monitoreo permanente, en tiempo real, de los focos incendiarios que amenazaban la zona, a través del Fire Information for Resource Management System, FIRMS (NASA 2017a), en combinación con imágenes satelitales VIIRS usando visualizador Worldview (NASA 2017b).

El día 30 de enero, se corroboró el control y extinción total de los focos incendiarios que amenazaban el cerro Los Robles, sin haber afectado el área desde donde fueron capturados los ejemplares de L. confusus. Finalmente, previa programación y alimentación de los ejemplares con tenebrionidos (Tenebrio molitor Linnaeus, 1758) durante los días 30 y 31 de enero, la reinserción de los ejemplares se hizo efectiva en terreno el día 01 de febrero del 2017, donde cada uno de ellos fueron liberados vivos, con aparente buen estado sanitario, en su georreferenciado lugar de origen.

Cabe destacar que durante estas actividades de terreno, se logró determinar una clara preferencia de la especie por zonas áridas, de pedregales amplios, con rocas de mediano o pequeño tamaño (20 a 50 $\mathrm{cm}$ aproximadamente), y casi nula o escasa cobertura vegetacional, con presencia de algunas gramíneas de baja altura, y uno que otro Quisco (Echinopsis chiloensis (Colla) H.Friedrich \& G.D.Rowley), Boldo (Peumus boldus Molina) o Quillay (Quillaja saponaria Molina), es decir, una formación vegetacional cercana a la definición de matorral xerófilo, pero con ausencia de espino (Acacia caven (Molina) Molina). Confirmamos también sus hábitos saxícolas, pero con alternancia a terrícolas. Considerando la superficie en la cual fueron obtenidos los 20 ejemplares, podemos establecer una abundancia simple de 0,8 a 4,6 individuos por hectárea para la especie en la cima del cerro Los Robles.

Este plan de rescate preventivo para L. confusus, cuyos objetivos fueron cumplidos a cabalidad, constituye una iniciativa pionera en Chile que debería ser considerada y sistematizada por las autoridades competentes en materias de protección de la diversidad biológica del país, ante la eventualidad futura de que nuevas catástrofes de origen natural o antrópico, amenacen la supervivencia de poblaciones de especies categorizadas como En Peligro o En Peligro Crítico de Extinción con reducidas áreas de distribución. 


\section{AGRADECIMIENTOS}

Al Servicio Agrícola y Ganadero, por el apoyo y rápida gestión para la emisión de la resolución de autorización de captura $\mathrm{N}^{\circ} 483 / 2017$. A todo el equipo de apoyo logístico, científico y de captura en terreno que participó en la ejecución de este plan: Alejandra Ponce, Beatriz Brito, Daniel Terán, Daniel Arias, Hernán Lorca, Jaime Troncoso-Palacios, Javiera Constanzo, Johanna Hodar, Nicole Sallaberry, Osvaldo Cabeza, Paola Henríquez, Paz Pérez y Violeta Barrera.

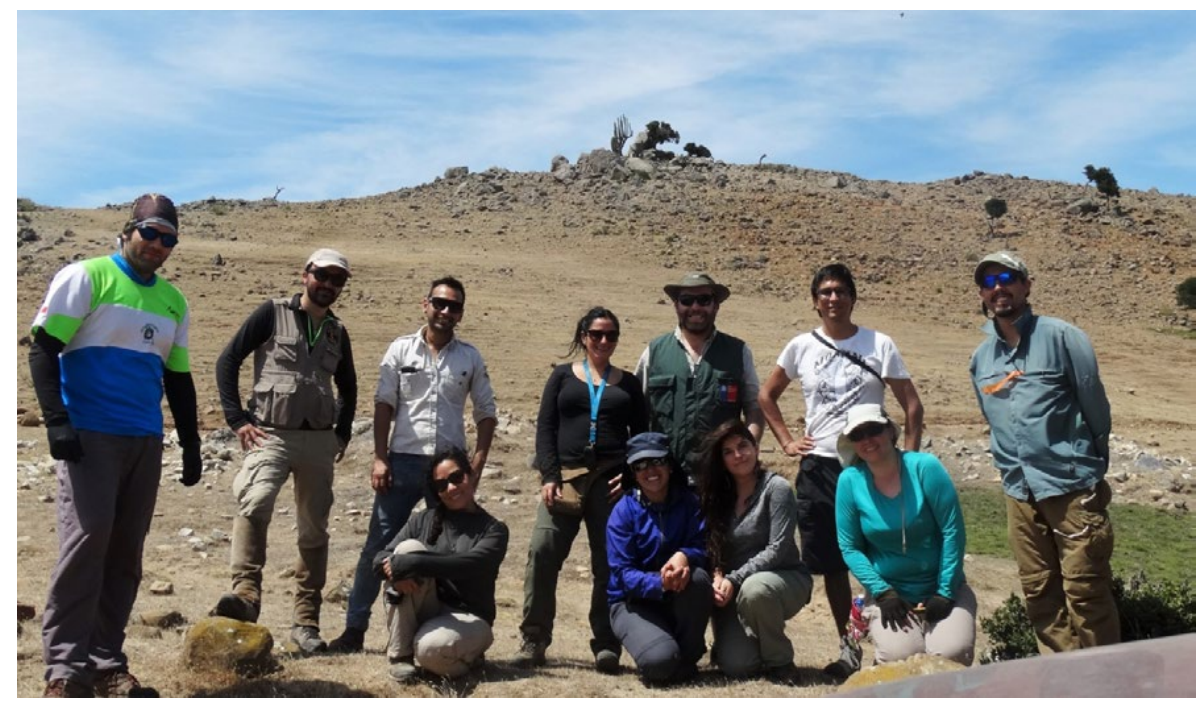

Figura 4. El equipo de rescate en terreno en la cima del cerro Los Robles.

\section{REFERENCIAS}

Arano, B., Herrero, P. \& Astudillo, G. (1999). Gestión genética de una especie amenazada: monitorización de la variación genética y cría en cautividad del lagarto gigante de El Hierro (Gallotia simonyi). Monografías de Herpetología 4: 85-100.

González-Gutiérrez, P.A. (2015). Área de distribución de Liolaemus confusus Núñez \& Pincheira-Donoso 2006, una especie endémica de la Región de O’Higgins. Boletín Chileno de Herpetología 2: 20-21.

Gual, F. (2009). Diseño del programa de manejo reproductivo del Cóndor de California (Gymnogyps californianus) en la Sierra de San Pedro Mártir, Baja California. Universidad Nacional Autónoma de México. Facultad de Medicina Veterinaria y Zootecnia. Informe Final. 48 pp.

Machado, A. (1985). Sinopsis del Plan de Recuperación del Lagarto Gigante de El Hierro. Bonner zoologische Beiträge 36: $471-480$
Ministerio del Medio Ambiente de Chile. (2014). Decreto Supremo $N^{\circ}$ 52/2014. $10^{\circ}$ Proceso de Clasificación según Reglamento de Clasificación de Especies del Ministerio del Medio Ambiente de Chile. Ficha de antecedentes de especie.

Ministerio del Interior y Seguridad Pública de Chile. (2017). Decreto $N^{\circ} 84 / 2017$. Declara estado de excepción constitucional de catástrofe en las Provincias de Colchagua y Cardenal Caro, de la Región de O'Higgins, y en las comunas de Vichuquén y Cauquenes, ambas de la región del Maule.

Ministerio de Agricultura de Chile. (2017a). Resolución Exenta $N^{\circ} 37 / 2017$. Declara situación de emergencia agrícola por los efectos de daño productivo derivados de incendios forestales que afectan a la Región del Libertador General Bernardo O'Higgins.

Ministerio de Agricultura de Chile. (2017b.) Informe de daño por incendios: Regiones desde Coquimbo hasta la Araucania, a fecha 29 de enero del 2017. 
NASA, National Aeronautic and Space Administration. (2017a). Fire Information for Resource Management System: https:/earthdata.nasa.gov/earth-observation-data/ near-real-time/firms, visualizado entre el 23 de enero y el 02 de febrero del 2017.

NASA, National Aeronautic and Space Administration. (2017b). Worldview: https://worldview.earthdata. nasa.gov/, visualizado entre el 23 de enero y el 02 de febrero del 2017.

Núñez, H. \& Pincheira-Donoso, D. (2006). Liolaemus confusus, una nueva especie de lagartija de la cordillera de la costa de Chile central (Sauria, Liolaminae): Evidencia fenética y citogenética. Boletín del Museo Nacional de Historia Natural de Chile 55: 75-86.
Ramírez-Álvarez, D., Salgado, I., Silva, P. \& Valdes, M. (2016). Nuevo registro y propuesta de ampliación del área de extensión y ocupación para Liolaemus confusus Núñez y Pincheira-Donoso, 2006 (Reptilia, Liolaemidae). Boletín del Museo Nacional de Historia Natural de Chile 65: 9-14.

Witzenberger, K.A. \& Hochkirch, A. (2011). Ex situ conservation genetics: a review of molecular studies on the genetic consequences of captive breeding programmes for endangered animal species. Biodiversity Conservation DOI 10.1007/s10531-011-0074-4. 
Este número ha recibido una ayuda del Vicerrectorado de Investigación y Transferencia de Conocimiento de la Universidad de Alicante
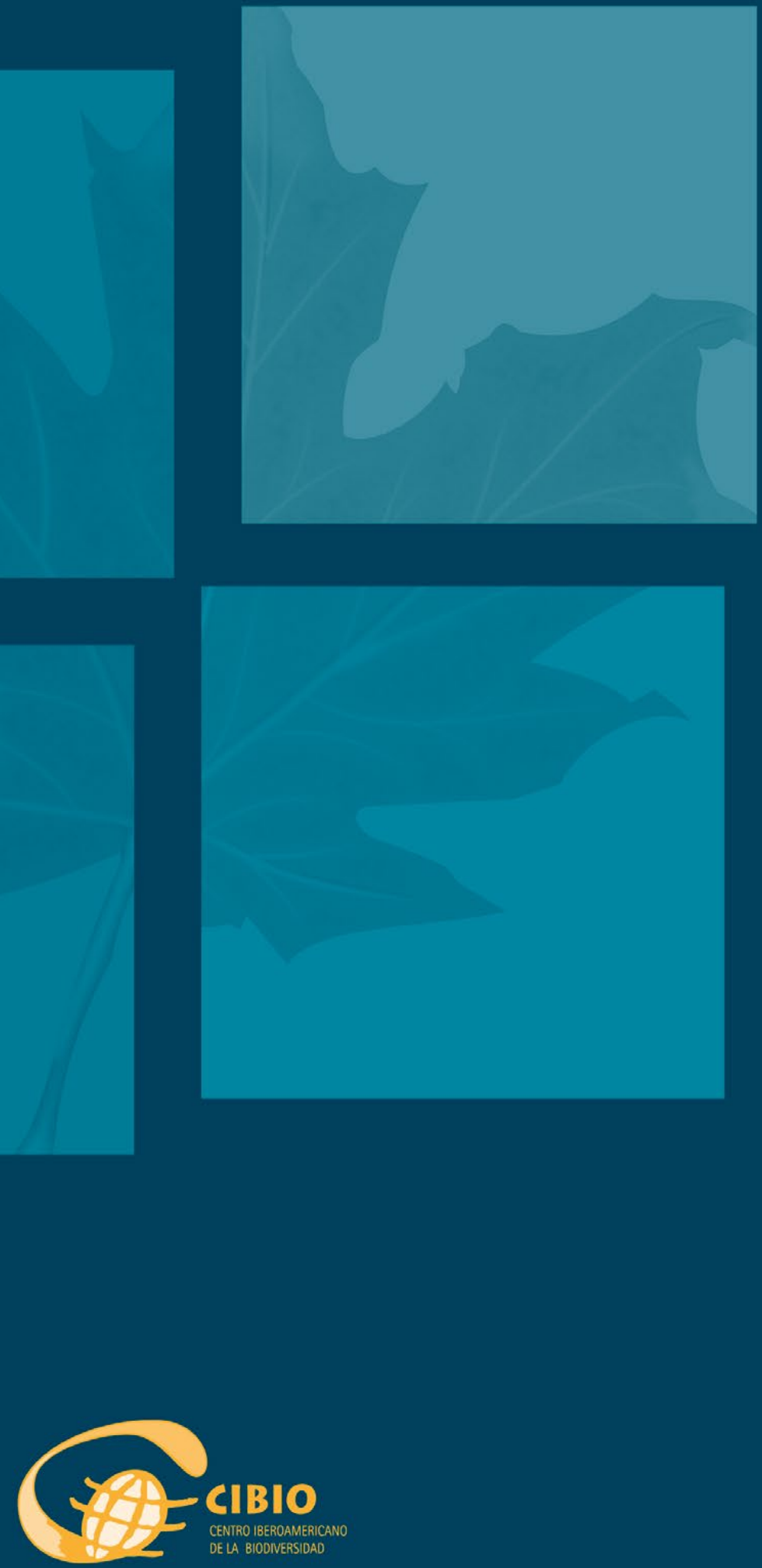

\section{Universitat d'Alacant
Universidad de Alicante}

